\title{
Hepatic Arterial Infusion Chemotherapy Is a Feasible Treatment Option for Breast Cancer with Liver-predominant Metastatic Disease
}

\author{
JUI-HU HSIAO ${ }^{1}$, HONG-TAI CHANG ${ }^{1}$, YEN-DUN TSENG ${ }^{1}$, CHIA-LING CHIANG ${ }^{2}$, \\ I-SHU CHEN $^{1}$, YU-CHIA CHEN ${ }^{1}$, PO-MING CHANG ${ }^{1}$ and BEING-WHEY WANG ${ }^{1}$ \\ ${ }^{1}$ Division of Gastroenterologic Surgery, Department of Surgery and ${ }^{2}$ Department of Radiology, \\ Kaohsiung Veterans General Hospital, Kaohsiung, Taiwan, R.O.C.
}

\begin{abstract}
Background: Patients with liver metastasis from breast cancer $(L M B C)$ are usually offered systemic therapy. However, for those with progressive liver disease and limited extra-hepatic conditions, local liver management becomes an option. Herein we present our experience with hepatic arterial infusion chemotherapy (HAIC). Patients and Methods: From 1999 to 2018, 42 patients with LMBC, who had progressive liver metastasis after systemic therapy, were treated with HAIC. A catheter was placed angiographically into the hepatic artery and remained there for 5 consecutive days. One cycle of chemotherapy consisted of mitoxantrone, 5-fluorouracil, folinic acid, and cisplatin. This treatment was repeated at monthly intervals. The medical records were reviewed and analyzed for hepatic tumor response, progression-free survival, overall survival and adverse effects. Results: Complete response was observed in two patients $(5 \%)$, partial response in 18 patients $(43 \%)$ and stable disease in eight patients (19\%). Fourteen patients (33\%) had progressive disease after HAIC. The median progression-free survival and overall survival were 8.4 and 19.3 months, respectively. There was no death related to HAIC. The patients with response to the treatment had a significant survival benefit $(p<0.005)$. Conclusion: HAIC can be an option for those with progressive liver disease who are heavily pretreated while their extra-hepatic conditions are minimal or stable.
\end{abstract}

This article is freely accessible online.

Correspondence to: Being-Whey Wang, MD, Division of General Surgery, Department of Surgery, Kaohsiung Veterans General Hospital, No. 386, Dazhong 1st Rd, Zuoying District, Kaohsiung 81362, Taiwan, R.O.C. Tel: +886 73422121 \#3085, Fax: +886 73468367, e-mail: rehookimo@gmail.com

Key Words: Liver metastasis, breast cancer, hepatic arterial infusion chemotherapy (HAIC), LMBC, heavily-pretreated patients.
Breast cancer is one of the most common types of cancer in the world ranking first in cancer-related incidence and second in death (1). Metastatic breast cancer is thought to be incurable. The common metastatic sites are bone, liver, lung, and brain. Median survival of patients with metastatic breast cancer is around 18-24 months $(2,3)$. Patients with metastasis may respond transiently to chemotherapy or endocrine therapy, but most of them exhibit progressive disease changes 1-2 years later (4). Metastatic liver disease may cause impairment of liver function and endanger the patient. Treatments for liver metastasis include systemic chemotherapy, endocrine therapy, surgical intervention, radiofrequency ablation, transcatheter arterial chemoembolization, and hepatic arterial infusion chemotherapy (HAIC) (5-9).

A five-day course of celiac artery infusion chemotherapy regimens including mitoxantrone, 5fluorouracil (5-FU), folinic acid, and cisplatinum was firstly reported by Beger et al., who claimed its effectiveness in preventing liver metastasis after pancreaticoduodenectomy for pancreatic cancer (10). These drugs have also been used in treating breast cancer, either for adjuvant or metastatic settings $(11,12)$. Since the report of Beger et al. in 1999, our Institute has performed HAIC with the Beger regimen for patients with heavily pretreated liver metastasis from breast cancer (LMBC) with or without extrahepatic metastasis. We present our results and compare with the reported series.

\section{Patients and Methods}

Study design. The local ethics committee (Human Research Committee of Kaohsiung Veterans General Hospital) was informed about the analyses, and approval was waived due to the retrospective nature of the study. All procedures followed were in accordance with the ethical standards of the responsible committee on human experimentation (institutional and national) and with the Helsinki Declaration of 1964 and later versions. 
Patients. From 1999 to 2018, 42 patients diagnosed with LMBC were treated with HAIC. Before that, most of them had received anthracycline-or taxane-based chemotherapy either for early breast cancer or after metastasis. Surgical resection and radiofrequency ablation of liver tumors were performed in seven and nine patients before HAIC, respectively. Trastuzumab or hormonal treatments were maintained with the treatment if the tumor was human epidermal growth factor receptor 2-positive (HER2+) or hormonal receptor-positive. The interval between the last treatments and HAIC were at least 1 month.

All patients were required to have appropriate hematological, serum aminotransferases, bilirubin, and renal function [absolute neutrophil count $(\mathrm{ANC}) \geq 1.5 \times 10^{9} / 1$, hemoglobin $\geq 8 \mathrm{~g} / \mathrm{dl}$ and platelet count $\geq 100 \times 10^{9} / 1$, alanine aminotransferase (ALT) and aspartate aminotransferase (AST) $\leq 5.0 \times$ upper limit of normal, bilirubin $\leq 2$ $\mathrm{mg} / \mathrm{dl}$, and serum creatinine $\leq 1.5 \mathrm{mg} / \mathrm{dl}]$. Liver metastasis was confirmed with computed tomography (CT) or magnetic resonance imaging (MRI)

HAIC regimen. A temporary catheter was introduced by the radiologist through the left subclavian artery and the tip of the catheter was placed in the proper hepatic artery. The patients received HAIC via an intra-arterial pump (13). Five courses were planned for each patient. One cycle consisted of $10 \mathrm{mg} / \mathrm{m}^{2}$ mitoxantrone on day $1,170 \mathrm{mg} / \mathrm{m}^{2}$ folinic acid directly followed by $600 \mathrm{mg} / \mathrm{m}^{2} 5$-FU on days 2 to 4 , and $60 \mathrm{mg} / \mathrm{m}^{2}$ cisplatinum on day 5 .

Evaluation and follow-up. Abdominal CT scan was the main evaluation tool for the tumor response. Analyses of response to treatment were based on the best response recorded during follow-up. The Response Evaluation Criteria in Solid Tumors (RECIST 1.1) were used for tumor measurement (14). Complete response (CR) was defined as the disappearance of all tumors; partial response (PR) was defined as a decrease of more than $30 \%$ in the sum of the longest diameters; progressive disease (PD) was defined as an increase of more than $20 \%$ in the sum of the longest diameters or other newly found lesions; stable disease (SD) was defined as neither CR, PR or PD.

Progressive-free survival (PFS) was defined as the time between the first HAIC and the day of diagnosis of PD. Overall survival (OS) was defined as the time between the first HAIC and the day of death.

Toxic analysis. All patients underwent standard clinical and laboratory examination including liver-related parameters at first presentation and during follow up after interventional treatment. The Common Terminology Criteria for Adverse Events (CTCAE) version 4.03 (National Cancer Institute, USA) (15) were used for toxicity assessments of laboratory values and clinical findings.

Statistical analysis. Statistical analysis was performed using SPSS (SPSS 21; IBM Corp., Armonk, NY, USA). Descriptive analysis of patient characteristics and findings was performed with continuous variables displayed as median with standard deviation or range and frequency data displayed as counts. The intergroup differences in categorical variables were analyzed by one-way ANOVA test. Survival (from first diagnosis, first diagnosis of liver metastases, first interventional treatment) was estimated according to the Kaplan-Meier method.

Possible factors influencing survival after first interventional LMBC treatment were included in a univariate Cox model. Multivariate analysis was performed by Cox proportional-hazard regression model. The log-rank test was used for survival comparison. Values of $p<0.05$ were considered statistically significant.

\section{Results}

Patient characteristics. A total of 42 patients with LMBC were enrolled on the protocol between February 1999 and March 2018. All of them were evaluable in respect of the treatment efficacy and safety. The median age was 47.5 years (range $=22-67$ years). Tumors were hormone receptor-positive in 32 patients $(76 \%)$ and HER2-positive in 12 (29\%) (Table I). Eighteen patients (43\%) had liver biopsy before HAIC. Postoperative adjuvant systemic therapy for primary breast cancer included anthracycline regimen such as epirubicin and cyclophosphamide (EC) or 5-FU and epirubicin and cyclophosphamide (FEC) for 40 patients (95\%), taxane for 39 patients $(93 \%)$, hormonal therapy for 32 patients $(76 \%)$ (Table II).

Twenty-one $(50 \%)$ patients had evidence of limited extrahepatic disease at the time of interventional LMBC treatment (bone metastases only, 11 patients; extrahepatic disease other than bone metastases, 10 patients). The mean number of liver metastases was 5.8 (range $=1-25)$. The diameter of the largest liver lesion ranged from $1-6.3 \mathrm{~cm}($ mean=2.3 cm).

All patients had received at least one line of chemotherapy for metastasis before HAIC (Table II). The median number of treatment lines was three with a range between one and five. Twenty-two (52\%) patients had received more than three lines of chemotherapy. Sixteen patients (38\%) had two lines of chemotherapy, and four $(10 \%)$ patients were treated with one line before HAIC. Three patients (7\%) were observed to have an elevated ALT level. The median interval from diagnosis of LMBC to presentation for HAIC was 5.5 (range=1-64) months. The Eastern Cooperative Oncology Group performance status was 0 in 39 patients (93\%), and 1 in three patients $(7 \%)$. The therapy agents used following HAIC due to disease progression are shown in Table III.

Treatment response. The median number of sessions of HAIC was 2.5 (range=1-6). Nine (21\%) patients received one session, and treatment discontinuation was due to disease progression in four patients, thrombosis of hepatic artery in one and refusal of further HAIC in four. The remaining 33 patients tolerated the first session well. Twelve patients received two sessions, seven patients had three, 13 had four and one patient had six.

Two patients (4\%) had CR and 18 patients $(43 \%)$ had PR. Eight patients (19\%) achieved SD and 14 patients $(33 \%)$ had progressive change after HAIC. There were no significant factors predicting which patients were responders (Table I).

PFS and OS. The median PFS was 8.4 months [range $=0.8-$ $78,95 \%$ confidence interval $(\mathrm{CI})=10.6-15.3$ months]. The 
Table I. Clinical characteristics of 42 patients with metastatic breast cancer.

\begin{tabular}{|c|c|c|c|c|c|}
\hline \multirow[b]{2}{*}{ Characteristic } & \multicolumn{5}{|c|}{ Response to HAIC } \\
\hline & Total & $\mathrm{CR}, \mathrm{PR}$ & SD & PD & $p$-Value \\
\hline Number & 42 & 20 & 8 & 14 & \\
\hline \multicolumn{6}{|l|}{ Age, years } \\
\hline Median (range) & $47.5(22-67)$ & $46(31-67)$ & $47.5(22-51)$ & $50.5(37-60)$ & 0.154 \\
\hline \multicolumn{6}{|l|}{ Original tumor status, n (\%) } \\
\hline $\mathrm{T} 1$ & $5(11 \%)$ & $3(15 \%)$ & $1(13 \%)$ & $1(7 \%)$ & 0.450 \\
\hline $\mathrm{T} 2$ & $25(60 \%)$ & $12(60 \%)$ & $4(50 \%)$ & $9(64 \%)$ & \\
\hline $\mathrm{T} 3$ & $10(24 \%)$ & $5(25 \%)$ & $3(37 \%)$ & $2(14 \%)$ & \\
\hline $\mathrm{T} 4$ & $2(5 \%)$ & $0(0 \%)$ & $0(0 \%)$ & $2(14 \%)$ & \\
\hline \multicolumn{6}{|c|}{ Original lymph node status, n (\%) } \\
\hline No & $11(26 \%)$ & $4(20 \%)$ & $3(37 \%)$ & $4(29 \%)$ & 0.770 \\
\hline N1 & $16(38 \%)$ & $7(35 \%)$ & $4(50 \%)$ & $5(35 \%)$ & \\
\hline $\mathrm{N} 2$ & $11(26 \%)$ & $6(30 \%)$ & $1(13 \%)$ & $4(29 \%)$ & \\
\hline $\mathrm{N} 3$ & $4(10 \%)$ & $3(15 \%)$ & $0(0 \%)$ & $1(7 \%)$ & \\
\hline \multicolumn{6}{|l|}{ Histologic type, n (\%) } \\
\hline Invasive ductal carcinoma & $38(90 \%)$ & $18(90 \%)$ & $8(100 \%)$ & $12(86 \%)$ & 0.360 \\
\hline Invasive lobular carcinoma & $2(5 \%)$ & $2(10 \%)$ & $0(0 \%)$ & $0(0 \%)$ & \\
\hline Other & $2(5 \%)$ & $0(0 \%)$ & $0(0 \%)$ & $2(14 \%)$ & \\
\hline \multicolumn{6}{|l|}{ Hormone receptor status, $\mathrm{n}(\%)$} \\
\hline Positive & $32(76 \%)$ & $17(85 \%)$ & $4(50 \%)$ & $11(79 \%)$ & 0.290 \\
\hline Negative & $10(24 \%)$ & $3(15 \%)$ & $4(50 \%)$ & $3(21 \%)$ & \\
\hline \multicolumn{6}{|l|}{ HER2 status, n (\%) } \\
\hline Positive & $12(29 \%)$ & $5(25 \%)$ & $2(25 \%)$ & $5(35 \%)$ & 0.110 \\
\hline Negative & $30(71 \%)$ & $15(75 \%)$ & $6(75 \%)$ & $9(64 \%)$ & \\
\hline \multicolumn{6}{|l|}{ Extrahepatic metastases, n (\%) } \\
\hline No & $21(50 \%)$ & $10(50 \%)$ & $3(37 \%)$ & $8(57 \%)$ & 0.140 \\
\hline Yes & $21(50 \%)$ & $10(50 \%)$ & $5(63 \%)$ & $6(43 \%)$ & \\
\hline \multicolumn{6}{|c|}{ Time from $\mathrm{BC}$ diagnosis to liver metastasis, months } \\
\hline Median (range) & $32.1(0-99)$ & $34.5(0-99)$ & $31.1(0-82)$ & $32.6(0-90.6)$ & 0.950 \\
\hline \multicolumn{6}{|c|}{ Time from liver metastases to first HAIC, months } \\
\hline Median (range) & $5.5(1-64)$ & $2.9(1-63)$ & $16.3(1-63)$ & $9.5(2-64)$ & 0.060 \\
\hline \multicolumn{6}{|l|}{ ALT before HAIC, n (\%) } \\
\hline$\leq \mathrm{ULN}$ & $39(93 \%)$ & $18(90 \%)$ & $7(87 \%)$ & $14(50 \%)$ & 0.200 \\
\hline$>\mathrm{ULN}-3 \times \mathrm{ULN}$ & $3(7 \%)$ & $2(10 \%)$ & $1(13 \%)$ & $0(0 \%)$ & \\
\hline \multicolumn{6}{|c|}{ Prior lines of chemotherapy, n (\%) } \\
\hline $1-2$ & $20(48 \%)$ & $12(60 \%)$ & $3(37 \%)$ & $5(35 \%)$ & 0.230 \\
\hline$\geq 3$ & $22(52 \%)$ & $8(40 \%)$ & $5(63 \%)$ & $9(64 \%)$ & \\
\hline \multicolumn{6}{|l|}{ Metastatic liver tumor size, n (\%) } \\
\hline$\leq 3 \mathrm{~cm}$ & $29(69 \%)$ & $13(65 \%)$ & $6(75 \%)$ & $10(71 \%)$ & 0.290 \\
\hline$>3 \mathrm{~cm}$ & $13(31 \%)$ & $7(35 \%)$ & $2(25 \%)$ & $4(29 \%)$ & \\
\hline \multicolumn{6}{|c|}{ Metastatic liver tumor number, n (\%) } \\
\hline$\leq 3$ & $17(40 \%)$ & $9(45 \%)$ & $2(25 \%)$ & $6(43 \%)$ & 0.140 \\
\hline$>3$ & $25(60 \%)$ & $11(55 \%)$ & $6(75 \%)$ & $8(57 \%)$ & \\
\hline \multicolumn{6}{|l|}{ Metastatic lobe of liver, n (\%) } \\
\hline Right/left & $20(48 \%)$ & $10(50 \%)$ & $3(37 \%)$ & $7(50 \%)$ & 0.800 \\
\hline Bilateral & $22(52 \%)$ & $10(50 \%)$ & $5(63 \%)$ & $7(50 \%)$ & \\
\hline
\end{tabular}

ALT: Alanine aminotransferase; BC: breast cancer; CR: complete response; HER2: human epidermal growth factor receptor 2; HAIC: hepatic arterial infusion chemotherapy; PD: progressive disease; PR: partial response; SD: stable disease; ULN: upper limit of normal.

median OS after first HAIC was 19.3 months (range $=3-112$ months, 95\% CI=14.7-23.8 months) (Figure 1). The median survival time from the initial diagnosis of breast cancer was 62.3 months and from diagnosis of liver metastasis was 23.8 months. The median OS was significantly longer in the responder group (26.7 versus 6.1 months, $p<0.001$; Figure 2 ). The median PFS was significantly longer in patients who had previously received fewer than three lines of systemic chemotherapy compared to those who received three lines or more (12.1 versus 5.6 months, $p=0.002$; Figure 3 ). The 
in vivo $32: 1635-1641(2018)$

Table II. Treatment received by patients with metastatic breast cancer prior to hepatic arterial infusion chemotherapy (HAIC).

\begin{tabular}{lrrccrrr}
\hline & \multicolumn{5}{c}{ Treatment } \\
\cline { 2 - 7 } Before HAIC & FEC & EC & FLC & Taxane & Trastuzumab & Hormonal & Other \\
\hline Yes & $35(83 \%)$ & $5(12 \%)$ & $2(5 \%)$ & $39(93 \%)$ & $12(29 \%)$ & $32(76 \%)$ & $23(55 \%)$ \\
No & $7(17 \%)$ & $37(88 \%)$ & $40(95 \%)$ & $3(7 \%)$ & $30(71 \%)$ & $10(24 \%)$ & $19(45 \%)$ \\
\hline
\end{tabular}

FEC: 5-Fluorouracil, epirubicin and cyclophosphamide; EC: epirubicin and cyclophosphamide; FLC: 5-fluorouracil, lipo-dox and cyclophosphamide.

median PFS and OS did not significantly differ between the patients with and those without extrahepatic lesions.

Univariate Cox regression regarding PFS, we identified fewer than three lines of prior systemic chemotherapy $(p=0.005)$ as a factor associated with a significantly favorable survival rate. In univariate and multivariate analyses of the OS, response of the liver tumors $(p<0.001)$ was found to be a significant prognostic factor. Other factors, such as age $(>45$ years), original breast tumor size $(>2 \mathrm{~cm})$, original number of lymph nodes involved ( $>3$ ), hormonal receptor status, HER2 status, presence of extrahepatic disease, size of metastatic liver tumors $(>3 \mathrm{~cm})$, number of metastatic liver tumor $(>3)$, and tumor markers (cancer antigen 15-3 $>30 \quad \mathrm{U} / \mathrm{ml}$ and carcinoembryonic antigen $>5 \mathrm{ng} / \mathrm{ml}$ ) had no significant impact on PFS or OS.

Nine patients were still alive at the time of writing. Six patients had died of lung metastasis, five of brain metastasis, and 22 of liver tumor progression.

Toxicity. The most common hematological toxicities were neutropenia $(11 \%)$, anemia $(2 \%)$, thrombocytopenia $(2 \%)$ and ALT elevation (10\%). Non-hematological toxicities included grade I-II anorexia (21\%). Two patients had neutropenic fever, two complained of abdominal pain, and one mentioned diarrhea. No treatment-related death was observed (Table IV).

\section{Discussion}

On account of the fact that most metastatic liver tumors are supplied by the hepatic artery, chemotherapeutic agents can be delivered through HAIC more specifically to malignant cells $(16,17)$. On the other hand, normal hepatocytes that mostly rely on the portal venous system are thus exposed to less chemotherapeutic agent. Therefore, HAIC provides a higher exposure to chemotherapy of malignant cells with minimized toxicities and the higher drug level may also overcome drug resistance. Indeed, chemoperfusion in cancer with liver metastasis has produced promising results for patients with colorectal cancer (18). However, the benefit of HAIC in patients with liver metastasis of breast cancer is not clear and
Table III. Treatment received by patients with metastatic breast cancer after hepatic arterial infusion chemotherapy $(N=23)$.

\begin{tabular}{lc}
\hline Agent & Number of patients \\
\hline Anthracycline & 3 \\
Taxane & 16 \\
Cisplatin & 7 \\
Lipo-dox & 3 \\
Vinorelbine & 6 \\
Eribulin & 2 \\
Lapatinib & 1 \\
Capecitabine & 12 \\
Avastin & 2 \\
Ixempra & 4 \\
Everolimus & 1 \\
5-Fluorouracil & 1 \\
\hline
\end{tabular}

Table IV. Toxicity experienced by patients treated with hepatic arterial infusion chemotherapy in this study. There were no grade 4 toxicities.

\begin{tabular}{lll}
\hline & \multicolumn{2}{c}{ Grade, n (\%) } \\
\cline { 2 - 3 } Toxicity & 2 & 3 \\
\hline Neutropenia & $1(2 \%)$ & $4(9 \%)$ \\
Anemia & $1(2 \%)$ & $1(2 \%)$ \\
Thrombocytopenia & 0 & 0 \\
Increase ALT/AST & $1(2 \%)$ & $3(7 \%)$ \\
Nausea/vomiting & $6(14 \%)$ & $3(7 \%)$ \\
Diarrhea & $1(2 \%)$ & 0 \\
Abdominal pain & $2(5 \%)$ & 0 \\
\hline
\end{tabular}

ALT: Alanine aminotransferase, AST: aspartate aminotransferase.

only a few retrospective analyses have been reported with use of intrahepatic infusion for breast cancer $(9,19-22)$. Whereas colorectal liver metastasis can be regarded as locoregional spread through portal circulation, other tumor types such as breast cancer with liver metastasis may only be the first site 


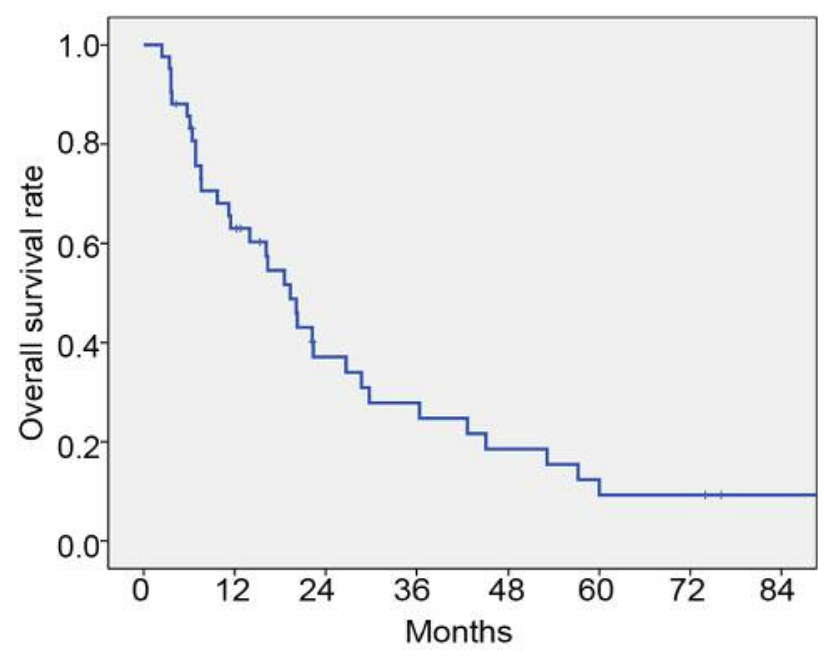

Figure 1. Kaplan-Meier curve of overall survival for all patients with metastatic breast cancer treated with hepatic arterial infusion chemotherapy. Median overall survival $=19.3 \quad(95 \%$ confidence interval=14.7-23.8) months.

of distant spread, denoting systemic hematogenous distant micro-metastases elsewhere. Clinically, approximately $50 \%$ of patients with breast cancer will develop distant metastases. Liver metastases are present in $15 \%$ of patients newly diagnosed with metastatic breast cancer $(23,24)$, and the liver is the only site of distant disease in one-third of these patients (25). Systemic chemotherapy or hormonal therapy is usually indicated for these patients.

In the present study, we performed HAIC for those patients with liver-predominant metastatic disease when further systemic chemotherapy was not available or deemed ineffective since all the patients had received anthracycline or taxane. The overall response rate was $48 \%$, including two cases of complete response, with an SD rate of $19 \%$. The resulting tumor control rate of $67 \%$ was better than the reported series, in which Maes et al. (22). and Tsimeridou et al. (26). found a response rate of $26.6 \%$ and $17.6 \%$ with SD rates of $16.7 \%$ and $35.3 \%$, respectively $(22,26)$. Recently, Tewes et al. also reported PR in $20 \%$ and SD in $38.6 \%$ with an HAIC protocol including mitomycin, 5-FU and melphalan (9).

Mitoxantrone is an effective agent for the treatment of advanced breast cancer with mild side-effects, especially with respect to nausea/vomiting, hair loss and cardiotoxicity (27). The literature also confirmed the feasibility of adjuvant therapy in combination with cyclophosphamide and fluorouracil for patients with early breast cancer. For the past decade, mitoxantrone has been used in the treatment of acute myeloid leukemia, hormone-refractory prostate cancer, and multiple sclerosis (28). While its mechanism of action remains incompletely described, it is thought that

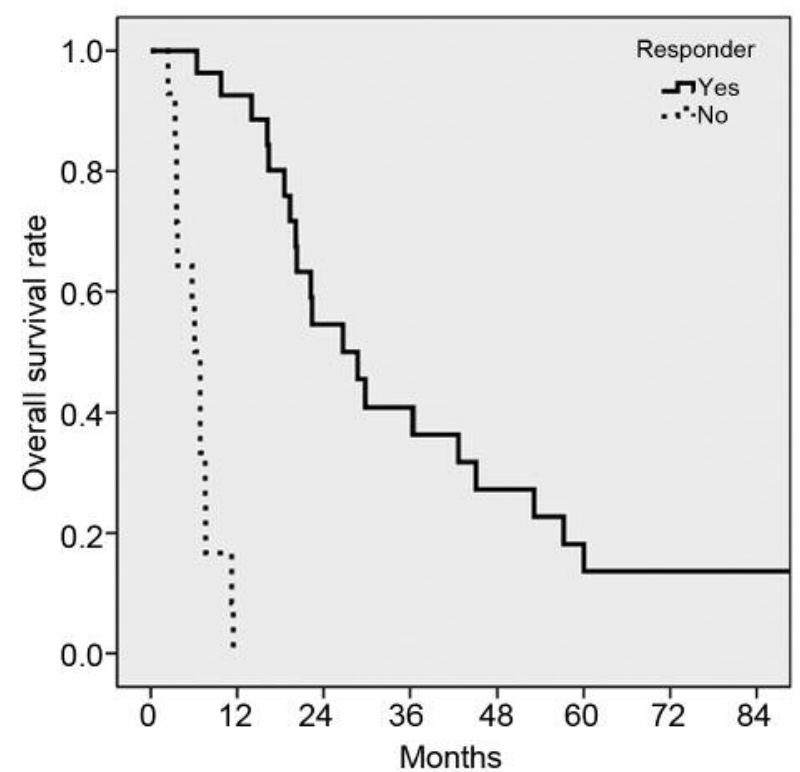

Figure 2. Kaplan-Meier curves of overall survival for patients with response (complete or partial response, stable disease) and for nonresponders (progressive disease) to treatment with hepatic arterial infusion chemotherapy. Median survival: Responders: 26.7 (95\% confidence interval=18.3-35.1) months; non-responders: 6.1 (95\% confidence interval=4.9-7.3) months; $p<0.001$. Median survival: Responders: 26.7 (95\% confidence interval=18.3-35.1) months; nonresponder: 6.1 (95\% confidence interval=4.9-7.3) months.

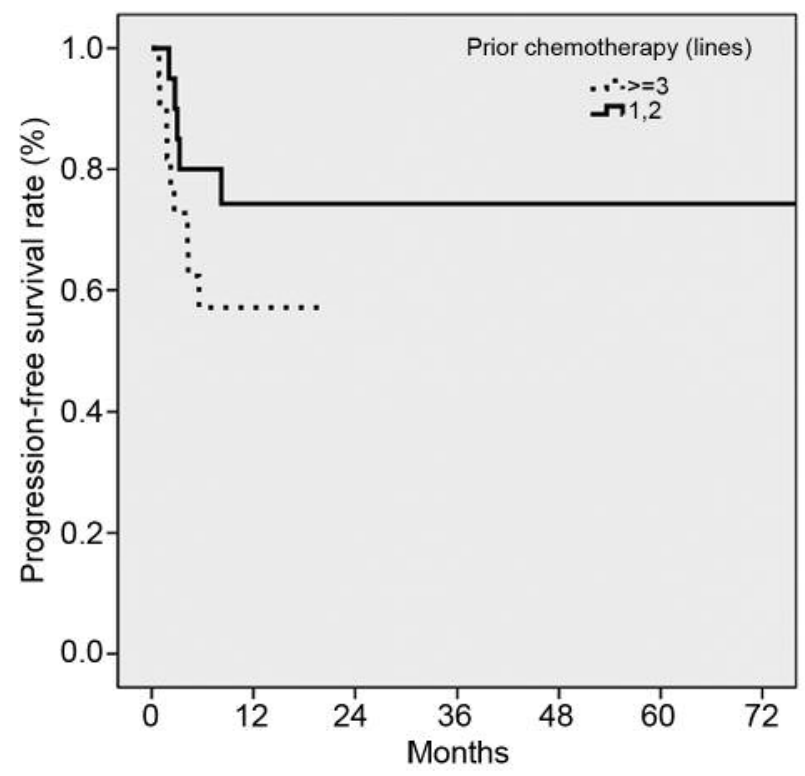

Figure 3. Kaplan-Meier curves of progression-free survival according to the number of lines of previous chemotherapy before treatment with hepatic arterial infusion chemotherapy. Median survival: Fewer than three lines: 12.1 (95\% confidence interval=6.6-17.6) months, more than three lines: 5.6 (95\% confidence interval=3.2-7.9) months; $p=0.002$. 
mitoxantrone intercalates with DNA resulting in DNA strand breaks. In addition, it has been characterized as a DNA type II topoisomerase inhibitor in a bacterial model $(29,30)$.

In a well designed in vitro study, it was demonstrated that mitoxantrone targets ubiquitin specific peptidase 11 (USP11), which is a deubiquitinating enzyme that works in concert with germ-line mutation of breast cancer 2 gene (BRCA2) to facilitate DNA homologous recombination by recruiting components of the DNA repair complex $(31,32)$. In this regard, mitoxantrone may be a good combination component. One of the components in our regimen for HAIC, cisplatin, interacts with DNA, preferentially binding nucleophilic N7 sites on purine bases (33). As a consequence, protein-DNA complexes and DNA-DNA inter-and intra-strand adducts are generated, inducing cytotoxicity. Cisplatin also increases the folate concentration in cancer cells, reinforcing the effect of 5FU through the formation of an inactive ternary complex (34, 35). Taken together, these preclinical studies showed synergistic chemotherapeutic effect in the regimen used for the present study. In the study of Lekakis et al., cisplatin and 5-FU were given with relative safety in the setting of liver dysfunction; many of their patients had liver impairment and were unable to receive other aggressive regimens due to the fact that the liver plays an important role in the metabolism of cytotoxic chemotherapeutics (36).

The choice for the less frequently used mitoxantrone was based on the study of Beger et al. (10), revealing tolerable toxicity after intra-arterial admission when combined with 5FU and cisplatinum. Marjolein et al. reported grade 3 and 4 leucopenia rates of 20 and $2 \%$, respectively, using the same protocol for adjuvant intra-arterial chemotherapy and radiotherapy in resectable pancreatic and periampullary cancer (37). The regimen for HAIC in present study was well tolerated and the toxicity profile was more favorable in comparison with mitomycin/5-FU regimens, in which substantial rates of hepatic and hematological adverse effects were found (9).

All patients in our study were heavily pretreated with a median of three previous chemotherapy regimens in treatment for early breast cancer. The median PFS was 8.4 months and median OS from introduction of HAIC was 19.3 months. These data were also better than other reported findings of PFS of 2 to 3 months and OS of 7 months $(9,22)$. Up to seven lines of systemic chemotherapy before HAIC reported in their studies may be the reason for this difference. Indeed, our analysis also showed that those having received three or more lines of systemic chemotherapy had a shorter PFS. Patients with response to HAIC treatment had a longer OS than those with progressive liver disease, indicating that control of the liver condition played an important role in these patients with liver-predominant metastatic disease.

The retrospective character is the main limitation of this study. The small number of patients was collected over a long period of time. This means that for a prospective randomized trial it may be difficult to recruit appropriate patients. During the study period, a number of advances have also been made in multiple areas of breast cancer treatment. In particular, systemic therapies have improved with the addition of the targeted biological agents such as pertuzumab and trastuzumab emtansine (T-DM1) to the treatment of patients with HER2positive tumors, and cyclin-dependent kinases 4 and 6 inhibitors to the treatment of patients with hormonal receptorpositive tumors. However, these biological agents may not be financially available for every patient.

\section{Conclusion}

Our study proved a viable treatment option for heavily pretreated patients suffering from liver-predominant breast cancer metastasis. The regimen proposed, is safe and may be beneficial in patients whose extra-hepatic conditions are minimal or stable.

\section{Conflicts of Interest}

The Authors declare that they have no conflict of interest in regard to this study.

\section{Acknowledgements}

The Authors express appreciation to the Research Center of Medical Informatics at Kaohsiung Veterans General Hospital for assistance in data processing.

\section{References}

1 Siegel RL, Miller KD and Jemal A: Cancer statistics, 2018. CA Cancer J Clin 68(1): 7-30, 2018.

2 Miller KD and Sledge GW Jr.: The role of chemotherapy for metastatic breast cancer. Hematol Oncol Clin North Am 13(2): 415-434, 1999.

3 Leung AM, Vu HN, Nguyen KA, Thacker LR and Bear HD: Effects of surgical excision on survival of patients with stage iv breast cancer. J Surg Res 161(1): 83-88, 2010.

4 Cristofanilli $\mathrm{M}$ and Hortobagyi GN: New horizons in treating metastatic disease. Clin Breast Cancer 1(4): 276-287, 2001.

5 BacalbaSa N, Balescu I, Dima S and Popescu I: Long-term survivors after liver resection for breast cancer liver metastases. Anticancer Res 35(12): 6913-6917, 2015.

6 Selzner M, Morse MA, Vredenburgh JJ, Meyers WC and Clavien PA: Liver metastases from breast cancer: Long-term survival after curative resection. Surgery 127(4): 383-389, 2000.

7 Wang M, Zhang J, Ji S, Shao G, Zhao K, Wang Z and Wu A: Transarterial chemoembolisation for breast cancer with liver metastasis: A systematic review. Breast 36: 25-30, 2017.

8 Eichler K, Jakobi S, Gruber-Rouh T, Hammerstingl R, Vogl TJ and Zangos S: Transarterial chemoembolisation (tace) with gemcitabine: Phase ii study in patients with liver metastases of breast cancer. Eur J Radiol 82(12): e816-822, 2013.

9 Tewes M, Peis MW, Bogner S, Theysohn JM, Reinboldt MP, Schuler M and Welt A: Hepatic arterial infusion chemotherapy for extensive liver metastases of breast cancer: Efficacy, safety 
and prognostic parameters. J Cancer Res Clin Oncol 143(10): 2131-2141, 2017.

10 Beger HG, Gansauge F, Buchler MW and Link KH: Intraarterial adjuvant chemotherapy after pancreaticoduodenectomy for pancreatic cancer: Significant reduction in occurrence of liver metastasis. World J Surg 23(9): 946-949, 1999.

11 Onyenadum A, Gogas H, Markopoulos C, Bafaloukos D, Aravantinos G, Mantzourani M, Koutras A, Tzorakoelefterakis E, Xiros N, Makatsoris T, Fountzilas G and Kalofonos HP: Mitoxantrone plus vinorelbine in pretreated patients with metastatic breast cancer. J Chemother 19(5): 582-589, 2007.

12 Llombart-Cussac A, Pivot X, Rhor-Alvarado A, Le Cesne A, Le Chevalier T, Tursz T and Spielmann M: First-line vinorelbinemitoxantrone combination in metastatic breast cancer patients relapsing after an adjuvant anthracycline regimen: Results of a phase ii study. Oncology 55(5): 384-390, 1998.

13 Liang HL, Huang JS, Lin YH, Lai KH, Yang CF and Pan HB: Hepatic arterial infusion chemotherapy for advanced hepatocellular carcinoma by placing a temporary catheter via the subclavian route. Acta Radiol 48(7): 734-740, 2007.

14 Schwartz LH, Litière S, de Vries E, Ford R, Gwyther S, Mandrekar S, Shankar L, Bogaerts J, Chen A, Dancey J, Hayes W, Hodi FS, Hoekstra OS, Huang EP, Lin N, Liu Y, Therasse P, Wolchok JD and Seymour L: RECIST 1.1-Update and clarification: From the RECIST Committee. Eur J Cancer 62: 132-137, 2016.

15 Kern P, Kalisch A, Kolberg HC, Kimmig R, Otterbach F, von Minckwitz G, Sikov WM, Pott D and Kurbacher C: Neoadjuvant, anthracycline-free chemotherapy with carboplatin and docetaxel in triple-negative, early-stage breast cancer: A multicentric analysis of feasibility and rates of pathologic complete response. Chemotherapy 59(5): 387-394, 2013.

16 Ridge JA, Bading JR, Gelbard AS, Benua RS and Daly JM: Perfusion of colorectal hepatic metastases. Relative distribution of flow from the hepatic artery and portal vein. Cancer 59(9): 1547-1553, 1987.

17 Ackerman NB: Experimental studies on the circulation dynamics of intrahepatic tumor blood supply. Cancer 29(2): 435-439, 1972.

18 Kemeny N, Daly J, Reichman B, Geller N, Botet J and Oderman $\mathrm{P}$ : Intrahepatic or systemic infusion of fluorodeoxyuridine in patients with liver metastases from colorectal carcinoma. A randomized trial. Ann Intern Med 107(4): 459-465, 1987.

19 Nielsen DL, Norgaard H, Vestermark LW, Pfeiffer P, Jensen BK, Nelausen KM, Bergenfeldt M, Hermann KL and Jensen BV: Intrahepatic and systemic therapy with oxaliplatin combined with capecitabine in patients with hepatic metastases from breast cancer. Breast 21(4): 556-561, 2012.

20 Hara S, Hashizume S, Itoyanagi N, Ayabe H, Tagawa Y, Tsuji H, Oka T, Yamamoto S, Shinguu H, Tou K, Tooyama H and Yamazumi K: A case of hepatic arterial infusion chemotherapy for multiple liver metastasis from breast cancer. Gan To Kagaku Ryoho 24(12): 1809-1812, 1997.

21 Onogawa S, Ito K, Nishimura K, Umeda Y, Kojima K, Imai R, Nakajima N, Nakagami K, Tohyama K, Suzuki M, Arai K and Muro H: [liver metastases from breast cancer 14 years after radical mastectomy; successful treatment with hepatic arterial infusion chemotherapy and systemic endocrine therapy - a case report]. Gan To Kagaku Ryoho 24(12): 1804-1808, 1997.

22 Maes T, Wildiers H, Heye S, Demey W, Maleux G, Neven P, Van Oosterom AT and Paridaens R: Intra-hepatic mitomycin c bolus infusion in the treatment of extensive liver metastases of breast cancer. Breast Cancer Res Treat 110(1): 135-142, 2008.
23 Zinser JW, Hortobagyi GN, Buzdar AU, Smith TL and Fraschini G: Clinical course of breast cancer patients with liver metastases. J Clin Oncol 5(5): 773-782, 1987.

24 Hoe AL, Royle GT and Taylor I: Breast liver metastases - incidence, diagnosis and outcome. J R Soc Med 84(12): 714-716, 1991.

25 Insa A, Lluch A, Prosper F, Marugan I, Martinez-Agullo A and Garcia-Conde J: Prognostic factors predicting survival from first recurrence in patients with metastatic breast cancer: Analysis of 439 patients. Breast Cancer Res Treat 56(1): 67-78, 1999.

26 Tsimberidou AM, Vaklavas C, Fu S, Wen S, Lim JA, Hong D, Wheler J, Naing A, Uehara C, Wallace M and Kurzrock R: Hepatic arterial infusion therapy in advanced cancer and liver-predominant disease: The md anderson experience. Hepatogastroenterology 60(127): 1611-1623, 2013.

27 Landys K, Borgstrom S, Andersson $\mathrm{T}$ and Noppa H: Mitoxantrone as a first-line treatment of advanced breast cancer. Invest New Drugs 3(2): 133-137, 1985.

28 Fox EJ: Mechanism of action of mitoxantrone. Neurology 63(12 Suppl 6): S15-18, 2004.

29 Pommier Y, Leo E, Zhang $\mathrm{H}$ and Marchand C: DNA topoisomerases and their poisoning by anticancer and antibacterial drugs. Chemistry \& Biology 17(5): 421-433, 2010.

30 Bellosillo B, Colomer D, Pons G and Gil J: Mitoxantrone, a topoisomerase ii inhibitor, induces apoptosis of b-chronic lymphocytic leukaemia cells. Br J Haematol 100(1): 142-146, 1998.

31 Deng S, Zhou H, Xiong R, Lu Y, Yan D, Xing T, Dong L, Tang E and Yang $\mathrm{H}$ : Over-expression of genes and proteins of ubiquitin specific peptidases (usps) and proteasome subunits (pss) in breast cancer tissue observed by the methods of rfdd-pcr and proteomics. Breast Cancer Res Treat 104(1): 21-30, 2007.

32 Schoenfeld AR, Apgar S, Dolios G, Wang R and Aaronson SA: Brca2 is ubiquitinated in vivo and interacts with usp11, a deubiquitinating enzyme that exhibits prosurvival function in the cellular response to DNA damage. Mol Cell Biol 24(17): 74447455, 2004.

33 Galluzzi L, Senovilla L, Vitale I, Michels J, Martins I, Kepp O, Castedo M and Kroemer G: Molecular mechanisms of cisplatin resistance. Oncogene 31(15): 1869-1883, 2012.

34 Scanlon KJ, Newman EM, Lu Y and Priest DG: Biochemical basis for cisplatin and 5-fluorouracil synergism in human ovarian carcinoma cells. Proc Natl Acad Sci USA 83(23): 8923-8925, 1986.

35 Kim R, Tanabe K, Inoue H and Toge T: Mechanism (s) of antitumor action in protracted infusion of low dose 5-fluorouracil and cisplatin in gastric carcinoma. Int J Oncol 20(3): 549-555, 2002.

36 Lekakis L, Tryfonopoulos D, Pistamatzian N, Panopoulos C, Koumakis G, Demiri S and Efremidis A: Salvage chemotherapy with cisplatin and 5-fluorouracil in metastatic breast cancer. Particular activity against liver metastases. Anticancer Res 32(5): 1833-1837, 2012.

37 Morak MJ, van der Gaast A, Incrocci L, van Dekken H, Hermans JJ, Jeekel J, Hop WC, Kazemier G and van Eijck CH: Adjuvant intra-arterial chemotherapy and radiotherapy versus surgery alone in resectable pancreatic and periampullary cancer: A prospective randomized controlled trial. Ann Surg 248(6): 1031-1041, 2008.

Received July 7, 2018

Revised July 31, 2018

Accepted August 6, 2018 